Research Article

\title{
ISAR Imaging Based on Multiple Measurement Vector Model Sparse Signal Recovery Algorithm
}

\author{
Junjie Feng (iD \\ School of Physics and Electrical Engineering, Liupanshui Normal University, Liupanshui, Guizhou, China \\ Correspondence should be addressed to Junjie Feng; fzy028@163.com
}

Received 13 May 2020; Accepted 17 June 2020; Published 13 July 2020

Guest Editor: Liangtian Wan

Copyright ( 92020 Junjie Feng. This is an open access article distributed under the Creative Commons Attribution License, which permits unrestricted use, distribution, and reproduction in any medium, provided the original work is properly cited.

A multiple measurement vector (MMV) model blocks sparse signal recovery. ISAR imaging algorithm is proposed to improve ISAR imaging quality. Firstly, the sparse imaging model is built, and block sparse signal recovery algorithm-based MMV model is applied to ISAR imaging. Then, a negative exponential function is proposed to approximately block L0 norm. The optimization solution of smoothed function is obtained by constructing a decreasing sequence. Finally, the correction steps are added to ensure the optimal solution of the block sparse signal along the fastest descent direction. Several simulations and real data simulation experiments verify the proposed algorithm has advantages in imaging time and quality.

\section{Introduction}

Due to the characteristics of long distance, all-weather and all-weather, the inverse synthetic aperture radar (ISAR) imaging technology has been widely used in military, civil, and other fields $[1,2]$. Generally, the wide-band signal is used to improve range resolution in ISAR imaging. In order to obtain a high azimuth resolution, it is necessary to improve the target rotation accumulation angle or increase the time interval of coherent processing. For a long coherent processing interval (CPI), complex motion compensation is needed; however, it is difficult to achieve real-time imaging. When the number of echo pulses is limited or the echo pulse is interfered strongly, it is difficult to achieve the effect. Therefore, the imaging algorithm based on short CPI is necessary.

The theory of compressive sensing (CS) uses a small amount of measurement information to reconstruct the original signal with a large probability through optimization, which is the research hot spot in the field of signal processing [3-6]. It has been used in ISAR imaging [7, 8], MIMO radar signal processing $[9,10]$, and radar parameter estimation [11-14]. Because the actual contour of the target in sky imaging background is smaller than imaging area, the scatters of the target have sparse structure compared with the imaging area. The traditional sparse ISAR imaging algorithm mainly considers the recovery of individual scatters. However, the scatters are not independent. Because the target is a whole, different parts of the target are connected together. The strong scatterers of ISAR target can occupy many resolution cells, which have usually clusters or blocks in the imaging region. In this case, the common sparse reconstruction algorithm cannot completely describe the characteristic of target.

The analysis shows that, by using the inherent structural characteristics of the signal, it can the improve signal reconstruction performance greatly. If the block property structure of ISAR target is exploited, the better recovery performance can be obtained. Two-dimensional patterncoupled sparse Bayesian learning (PC-SBL) algorithm is proposed in [15]. However, the algorithm is computational expensive. One block sparse reconstruction algorithm is proposed to reconstruct the scattering coefficient of the target in the paper.

At present, most of the applications of sparse signal recovery algorithm in ISAR imaging are based on the single measurement vector (SMV) model, in which the ISAR echo signals are divided according to the distance unit and then the image can be obtained by combining the reconstructed result of each distance unit. However, ISAR imaging 
reconstruction algorithm based on the SMV model has low efficiency and poor real-time performance. The compressive sensing multiple measurement vector model can repeat observations of the information, and the MMV model can obtain better performance and improve the sparse signal reconstruction efficiency compared with that of the SMV model.

In order to improve ISAR imaging quality, a two-dimensional sparse signal reconstruction algorithm of ISAR imaging based on MMV model is proposed. All onedimensional range images are written together as a whole to be recovered by utilizing the potential block characteristics of ISAR target. The algorithm can not only improve the efficiency of reconstruction but also improve the quality of ISAR imaging.

The structure of the article is as follows: Section 2 introduces necessary ISAR imaging model and sparse signal recovery ISAR imaging algorithm. In section 3, the block sparse recovery algorithm for ISAR imaging algorithm based on MMV model is introduced in detail. Simulation and real data imaging results of ISAR are presented in section 4 . Finally, section 5 provides the conclusion.

\section{ISAR Imaging Model}

ISAR uses the relative motion of target and radar to obtain high resolution azimuth. Assuming the LFM signal transmitted by the radar is

$$
y(t)=\operatorname{rect}\left(\frac{t}{T_{a}}\right) \exp \left[j 2 \pi\left(f_{c} t+\frac{1}{2} \lambda t^{2}\right)\right],
$$

where $t$ is the fast time, $f_{c}$ expresses the carrier frequency, $\gamma$ is the chirp rate, and $T_{a}$ indicates the pulse duration, the backscattered ISAR signal can be written as

$$
\begin{aligned}
s(t)= & A \cdot \operatorname{rect}\left(\frac{t}{T_{a}}\right) \cdot \operatorname{rect}\left(\frac{t}{T}\right) \cdot \exp \left\{j 2 \pi \left(\left(t-\frac{2 R(t)}{c}\right)\right.\right. \\
& \left.\left.+\frac{1}{2} \gamma\left(t-\frac{2 R(t)}{c}\right)^{2}\right)\right\},
\end{aligned}
$$

where $c$ is the speed of light, $T$ is the CPI, and $A$ is the signal amplitude. After the distance pulse is compressed, the received signal can be expressed as

$$
\begin{aligned}
s(t)= & A \cdot \sin c\left[T_{a} \gamma\left(t-\frac{2\left(R_{0}+y\right)}{c}\right)\right] \cdot \exp \left[-j 4 \pi \frac{\left(R_{0}+y\right)}{\lambda}\right] \\
& \cdot \operatorname{rect}\left(\frac{t}{T}\right) \cdot \exp \left[-j 2 \pi\left(f \cdot t+\frac{1}{2} \beta \cdot t^{2}\right)\right],
\end{aligned}
$$

where $\lambda$ is the wavelength, $f$ is the Doppler frequency, and $\beta$ is change rate of Doppler frequency. It is assumed that there are $K$ strong scattering centers in a certain distance unit and the time-varying scattering can be ignored in short coherence processing; it can be expressed as

$$
y(t)=\sum_{k=1}^{K} x_{k} \cdot \operatorname{rect}\left(\frac{t}{T}\right) \cdot \exp \left(-j 2 \pi f_{k} t\right)+n,
$$

where $x_{k}$ and $f_{k}$ are the $k^{\text {th }}$ scattering centers' complex coefficient and Doppler frequency, respectively, and $n$ is the noise, The time sequence can be expressed as $t=[1: N]^{T} \cdot \Delta t$, where $\Delta t=\left(1 / f_{r}\right)$ is the time interval and $f_{r}$ is the pulse repetition frequency. $N=(T / \Delta t)$ is the number of pulses. $\Delta f_{d}$ is the Doppler frequency resolution interval, and the sparse Doppler sequence is $f_{d}=[1: M] \cdot \Delta f_{d}$, where $M=\left(f_{r} / \Delta f_{d}\right)$ and $M$ is the number of Doppler unit corresponding to $\Delta f_{d}$. So, construct the basis matrix as $\Psi=\left\{\phi_{1}, \phi_{2}, \ldots, \phi_{m}, \ldots, \phi_{M}\right\}$, $\varphi_{M}(t)=\exp \left(-j 2 \pi f_{d}(m) t\right), 0<m \leq M$. The discrete signal equation received by ISAR can be expressed as

$$
y=\Phi w+n
$$

where $w$ is the coefficient vector composed of the reflection coefficient and $n$ is the noise vector.

Because $w$ contains the information of the strong scattering point of the target, it is a sparse vector. The solution of $w$ is equivalent to the following optimization problem:

$$
\widehat{w}=\arg \min \|w\|_{P} \quad \text { subject to }\|\mathrm{y}-\Phi w\|_{2}<\eta,
$$

where $\eta$ is a small constant, and the value is related to noise variance; $P$ is the Lp norm.

According to the above optimization, the signal of each distance unit is recovered and then combined together to obtain the ISAR image. Because every distance element needs to be recovered, it will increase the computational burden and affect the imaging efficiency. Based on this, the paper studies the ISAR imaging model based on MMV. If all one-dimensional range profiles are written together as a whole, the total received signal can be expressed as

$$
Y=\Phi W+N \text {, }
$$

where $W$ is the target scatter point coefficient matrix, which is the ISAR image.

In the traditional MMV model, the nonzero signals in each column of matrix $W$ have the same supporting set. For the equation (7), the scatter points of distance unit have different supporting set. Because the scatter points of ISAR targets have the characteristics of cluster or block distribution, the imaging result can be obtained by the following block L0 sparse signal recovery algorithm:

$$
\min _{w}\|W\|_{0} \quad \text { s.t. }\|Y(:, n)-\Phi W(:, n)\|<\eta \quad n=1, \ldots N,
$$

where $\|W\|_{0}$ expresses a block sparse L0 norm of a matrix, $\eta$ is a small positive number relating with noise, and $N$ is the number of columns of $W$.

The smoothed function $G_{\sigma}(w)=N-\sum_{i} \exp \left(-w_{i}^{2} / \sigma\right)$ approaches the L0 norm when the parameter $\sigma$ approaches zero [16]. When the parameter $\sigma \longrightarrow \infty, G_{\sigma}(w)$ approximates the L2 norm. Therefore, the algorithm does not search for sparse solution because L2 norm cannot describe 


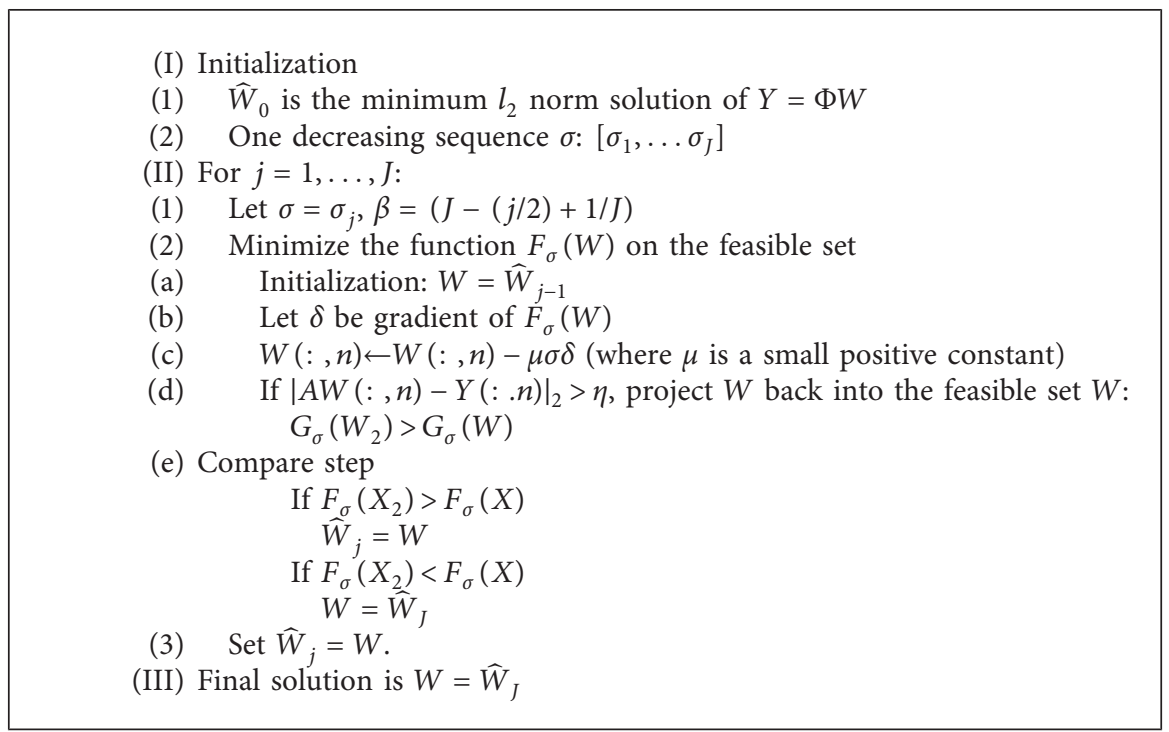

Algorithm 1: The block SL0 sparse signal recovery algorithm based on MMV model.

sparsity. A negative exponential function $F_{\sigma}(w)=N-\sum_{i} \exp \left(-\left|w_{i}\right| / \sigma\right)$ as smoothed function is proposed to obtain an approximate L0 norm solution. When the parameter $\sigma \longrightarrow \infty, F_{\sigma}(w)$ approaches L0 norm. Thus, the sparse solution can be obtained by using the new function at the very beginning of iteration.

For the one-dimensional block discrete signal $w$, the block structure is expressed as follows:

$$
w=\left[w_{1}, \ldots, w_{d}, \ldots, w_{2 d}, \ldots, w_{P d}\right],
$$

where $P$ is the size of the block and $d$ is the number of blocks. The block smoothed L0 function can be expressed as

$$
F_{\sigma}(w)=P-\sum_{P=1}^{P} g_{\sigma}\left(\sqrt{\sum_{i=1}^{d}|w((P-1) d)+i|^{2}}\right) .
$$

\section{MMV Block Sparse Signal ISAR Imaging Algorithm}

In the double-loop-layer SL0 algorithm [16], the solution of $\sigma=\sigma_{j}$ is only the initial value when $\sigma=\sigma_{j+1}$. So, it is not necessary to solve the precise solution of $\sigma=\sigma_{j}$. According to this observation, the inner loop layer is canceled, at the same time, the step size in the out layer is decreased. It means that the searching density of the variable parameter $\sigma$ is increased. For every $\sigma_{j}$, only one fastest descent searching is used to solve the minimization solution of the function $F_{\sigma_{j}}(w)$. The proposed algorithm ensures the block reconstruction accuracy, and the computation amount does not increase. The steepest descent method should reduce the cost function in every step, but it is not necessarily the descent direction in the actual solution process. Therefore, for the above algorithm, the step of checking whether the solution is descending is added in each iteration. If it is not along descent direction, the midpoint of the previous point and the current point is used to ensure that the search direction follows the fastest descent direction. The proposed algorithm is called improved MMB block sparse smoothed L0 algorithm (simplified as MBSSL0). The high ISAR imaging algorithm based on MMV mode is expressed as Algorithm 1.

If the selected step size is large, the algorithm will not converge. If the selected step size is too small, it will affect the convergence speed and reduce the calculation efficiency. Therefore, a larger step size is selected in the initial search because the search point is far from the minimum value point. When the search point is gradually close to the minimum value, the search step size should be gradually reduced. $J$ is the iterative loop number, and $\sigma_{J}$ should be chosen as less than the minimum value of the sparse solution. Letting $\mu=\beta \max |X| / L_{0}$ guarantees that it moves only a small part of the maximum value.

\section{Simulation Results}

4.1. One-Dimensional Block Spike Signal Recovery. The signal model is $y=A w+n$, and the sparse matrix $A$ is $80 \times 160$. Each element of $A$ is Gaussian distributed. wis a block sparse signal, whose nonzero block coefficients are uniform \pm 1 . The block size is $8 . n$ is the independent Gaussian random vector. The performance using BOMP [17], BCoSaMp [18], BSL0 [19], BSPG L1 [20], and MBSSL0 are compared. For BSL0 algorithm, the number of outer loops and inner loops is 20 and 10, respectively. For BSSL0 algorithm, the loop number is 200. Define minimum mean absolute value error MAE $=10 \log _{10}(\|x-\hat{x}\| / N)$, where $x$ is the true solution and $\hat{x}$ is the estimation value. The experiment was repeated 500 times. The computational times, correct position reconstruction frequencies, and MAE are averaged.

Figure 1 shows the average computational times with different block sparsity by different algorithms. We can see that BOMP, BSL0, and MBSSL0 algorithms have less computation than the other algorithms. The correct position 


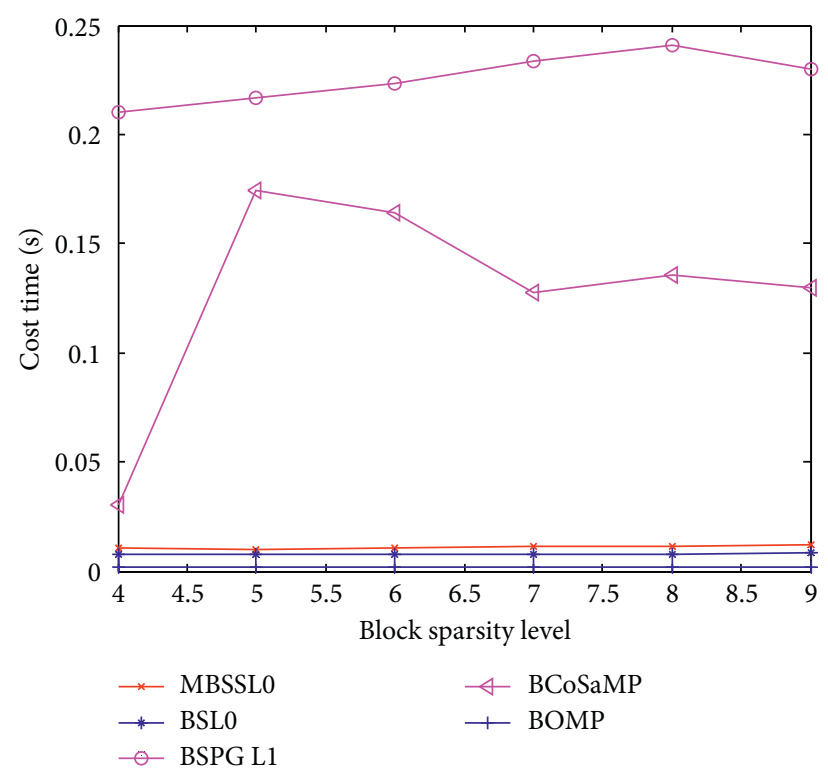

Figure 1: Computational costs for different algorithms with block sparsity.

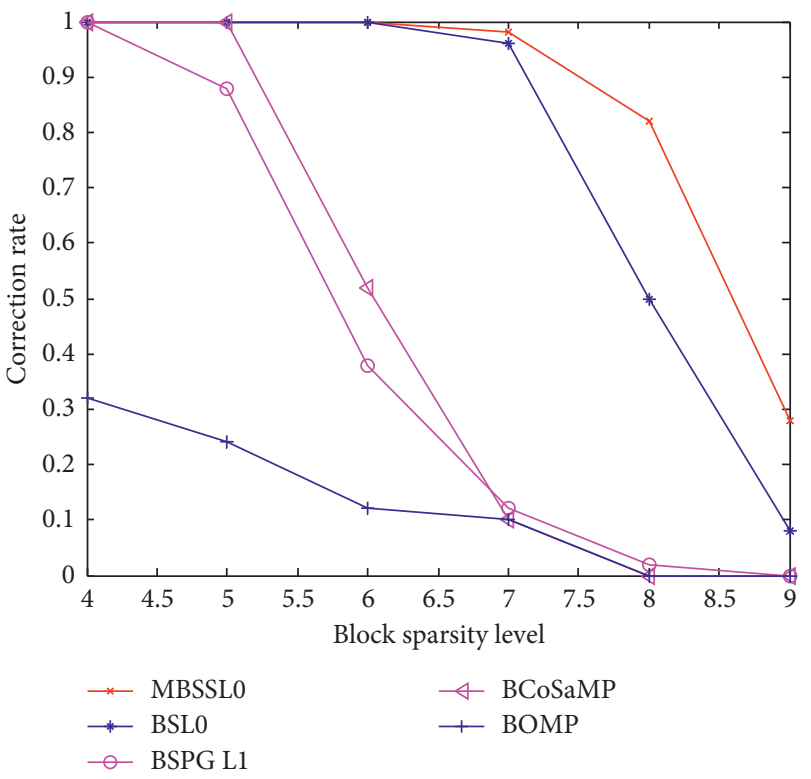

Figure 2: Correct estimation for different algorithms with block sparsity.

estimation and MAE with different block sparsities for different algorithms are shown in Figures 2 and 3. We can see that the performances of MBSSL0 algorithm are competitive with other algorithms.

4.2. ISAR Imaging Using Real Data. The real data are the measured echo data of yak-42 aircraft from the ISAR system. The radar parameters are as follows: the signal frequency band width $400 \mathrm{MHz}$, the center carrier frequency $10 \mathrm{GHz}$, and the data pulse repetition frequency $100 \mathrm{~Hz} .256$ echo pulses are selected in the simulation. Figures 4-6 show the imaging results obtained by these algorithms using 16, 32, and 64 pulses that are randomly chosen from 256 pulses. The uniform block is used in the block sparse reconstruction. The ISAR image is divided as $4 \times 2$ small blocks, where 4 is 4 cross-range units. The loop number of algorithm is 200 . The simulation results are compared visually and quantitatively with those images obtained by some sparse signal recovery methods including MOMP algorithm [21], SBL algorithm [22], PC-SBL algorithm, and MBSSL0 algorithm in this paper, respectively. The recovery images of MBSSL0 algorithm show more information in the neck of plane when pulse number is 16 . The images obtained by MBSSL0 algorithm are similar to PC-SBL algorithm when the plus numbers are 32 and 64 . The imaging results of MBSSL0 algorithm generate better visual quality, and the ISAR images are more intensive than MOMP algorithm and MSBL. 


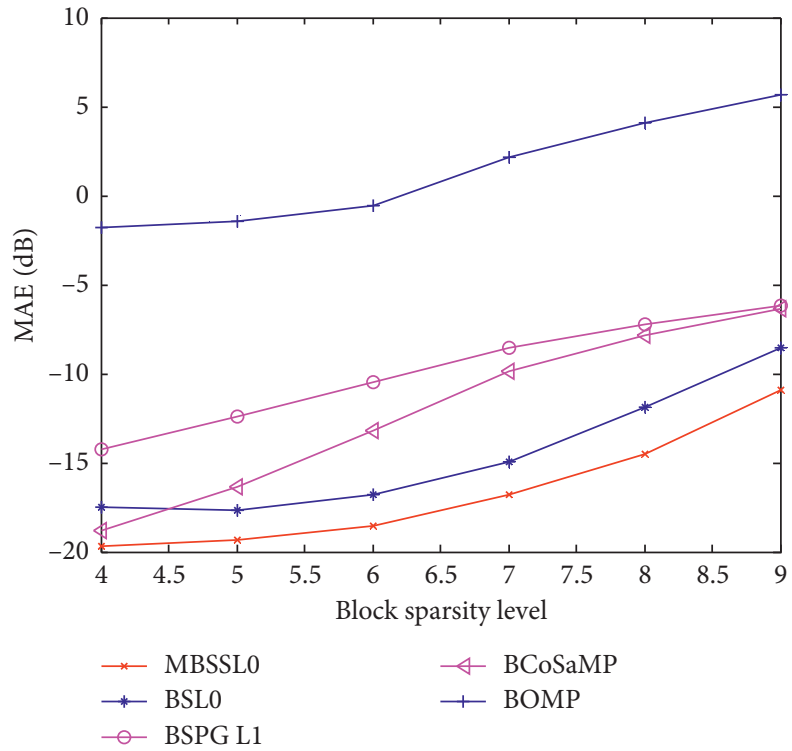

FIgURE 3: MAE for different algorithms with block sparsity.

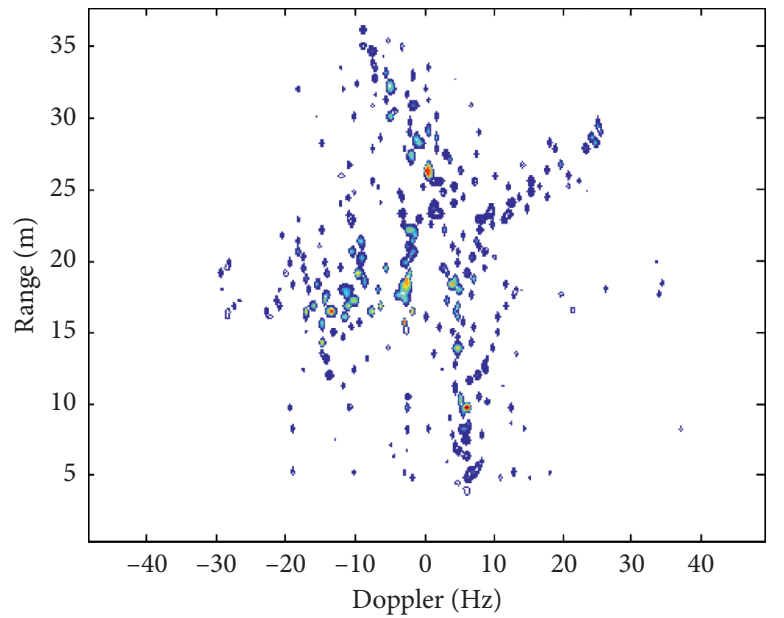

(a)

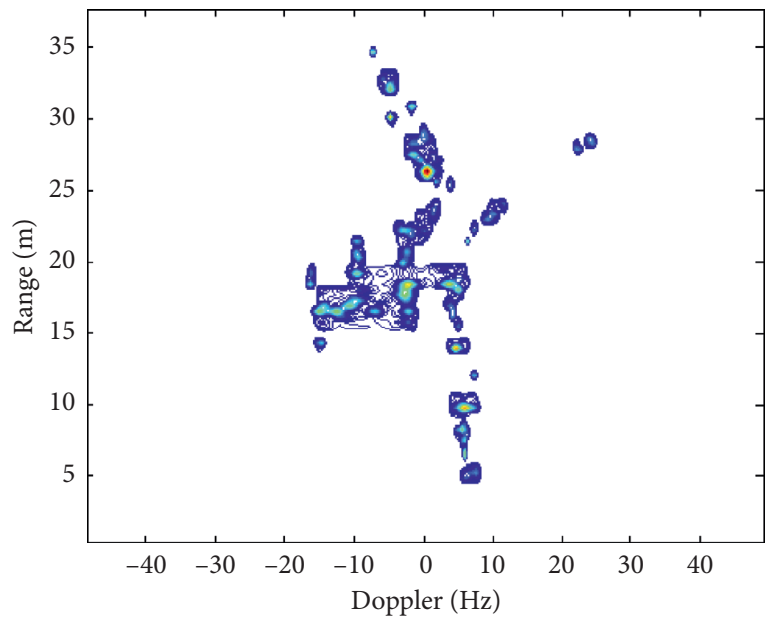

(c)

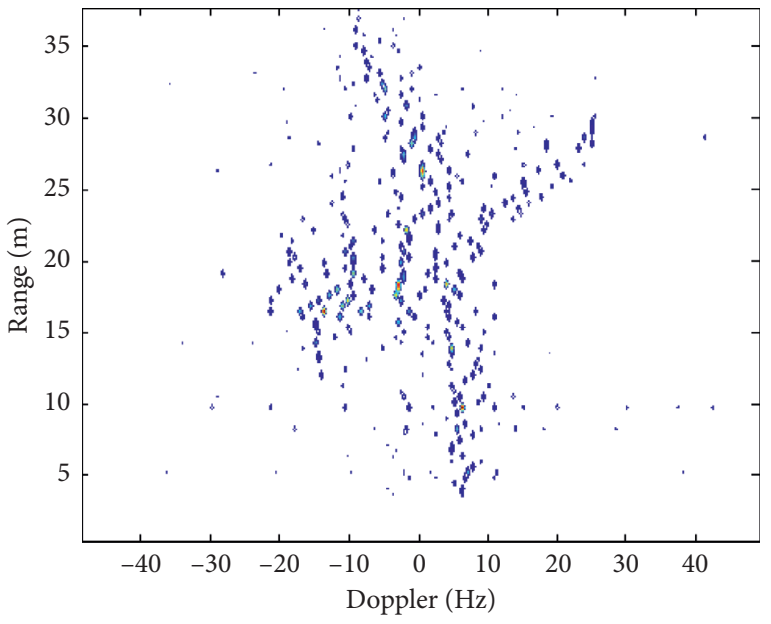

(b)

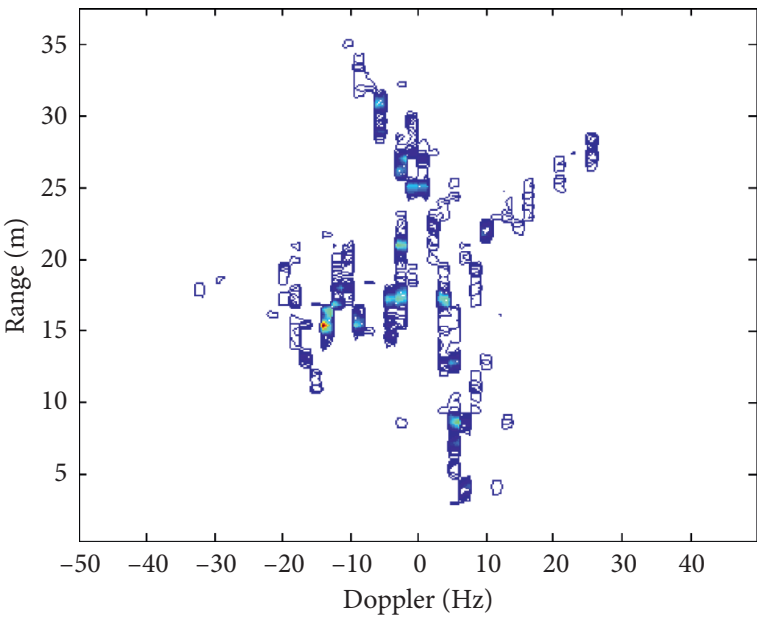

(d)

Figure 4: Reconstructed images using 16 pulse numbers: (a) MOMP, (b) MSBL, (c) PC-SBL, and (d) MBSSL0. 


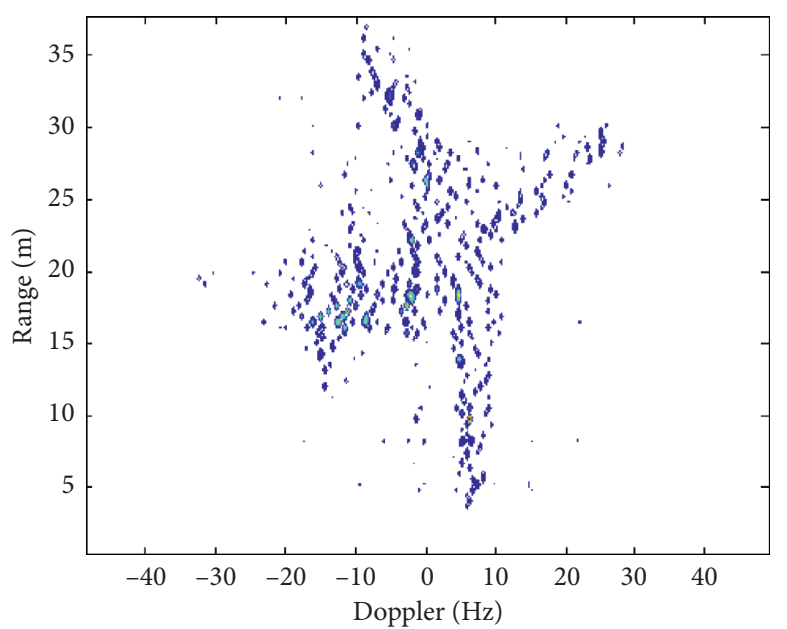

(a)

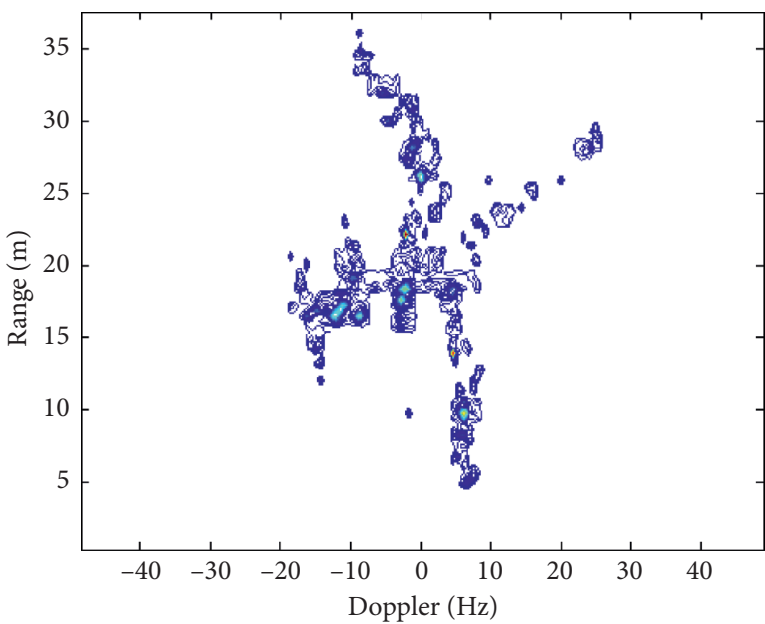

(c)

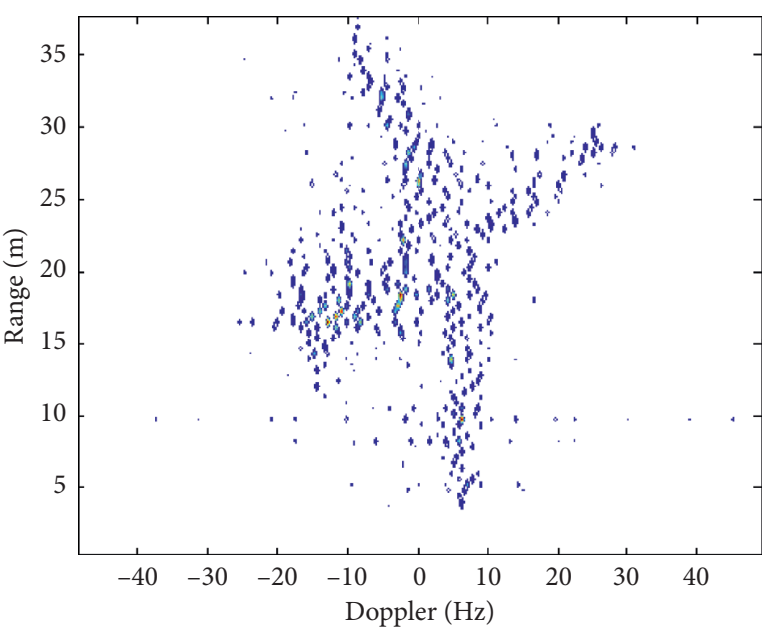

(b)

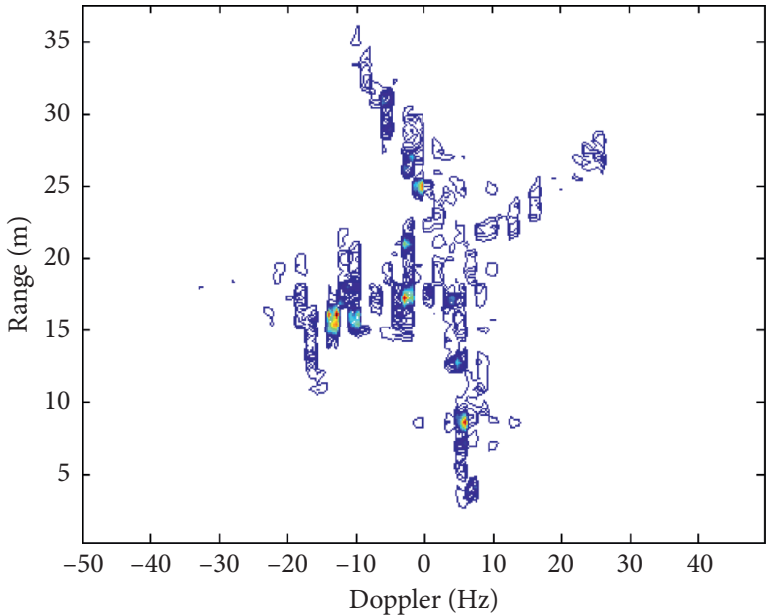

(d)

Figure 5: Reconstructed images using 32 pulse numbers: (a) MOMP, (b) MSBL, (c) PC-SBL, and (d) MBSSL0.

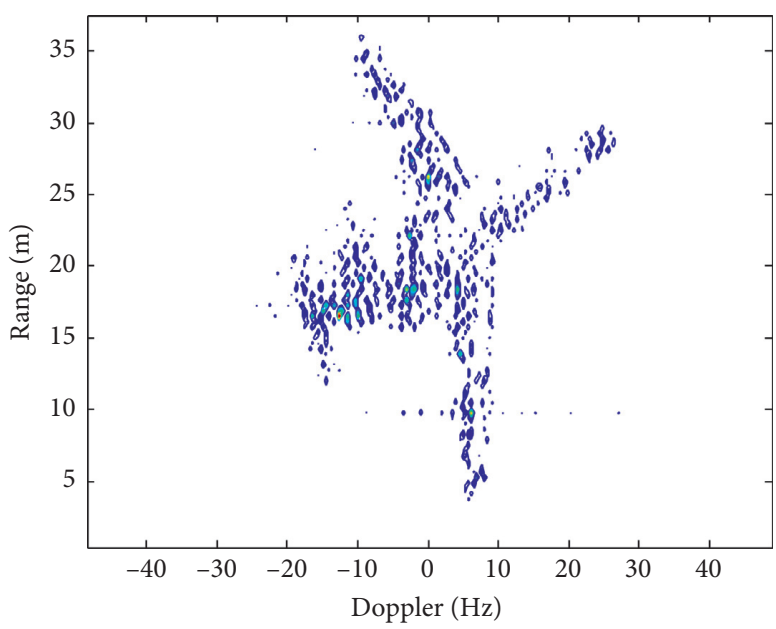

(a)

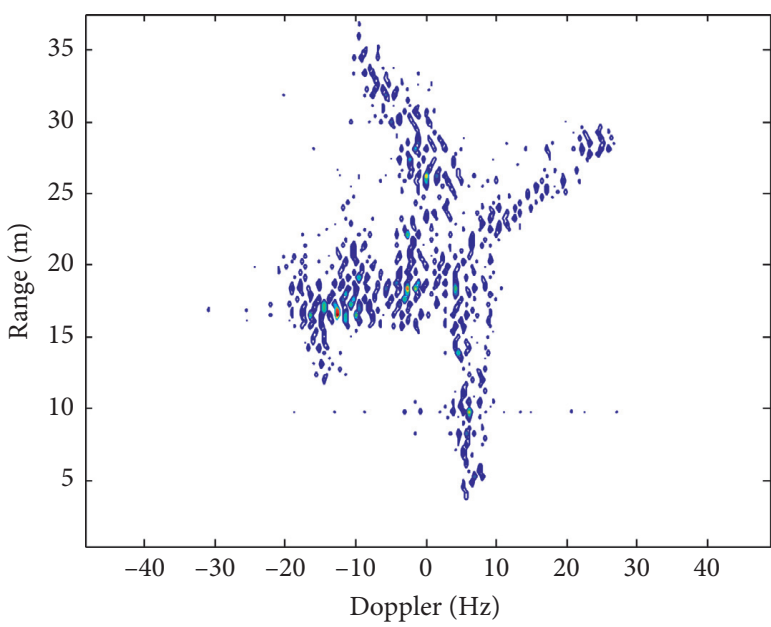

(b)

Figure 6: Continued. 


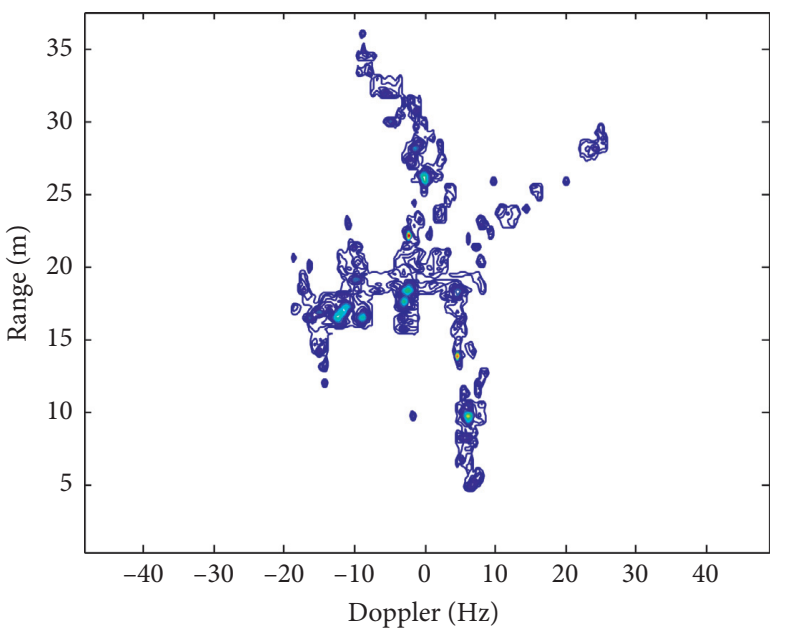

(c)

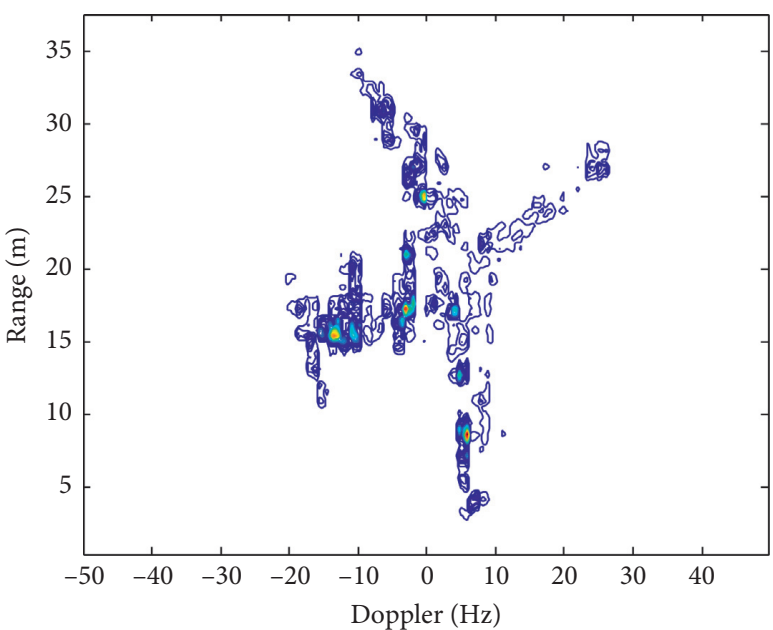

(d)

Figure 6: Reconstructed images using 64 pulse numbers: (a) MOMP, (b) MSBL, (c) PC-SBL, and (d) MBSSL0.

TABle 1: Average running times of the algorithm.

\begin{tabular}{lcccc}
\hline Algorithm & MOMP & MSBL & PC-SBL & MBSSL0 \\
\hline Run time (sec) & 97 & 172 & 228 & 102 \\
\hline
\end{tabular}

Considering the block sparsity, MBSSL0 algorithm can describe the characteristics of the signal better and has better signal reconstruction performance. The computation time using different algorithms are provided when the pulse number is 32 in Table 1 . It can be seen that the MBSSL0 algorithm saves computational time.

\section{Conclusion}

In this paper, one ISAR imaging algorithm of MMV block sparse reconstruction is proposed by considering the block structure of ISAR target in the imaging scene. Based on the recovery algorithm of smoothed block L0 norm, a revised step is added to ensure that the optimal solution is searched along the direction of the fastest descent. Experimental results show that the algorithm can save imaging time and improve ISAR imaging quality effectively with fewer pulses.

\section{Data Availability}

The data used to support the findings of this study are included within the article.

\section{Conflicts of Interest}

The author declares no conflicts of interest.

\section{Acknowledgments}

This work was partially supported by the National Science Foundation of China (no. 61471191).

\section{References}

[1] Y. Chen, Q. Zhang, N. Yuan, Y. Luo, and H. Lou, "An adaptive ISAR-imaging-considered task scheduling algorithm for multi-function phased array radars," IEEE Transactions on Signal Processing, vol. 63, no. 19, pp. 5096-5110, 2015.

[2] L. Zhao, L. Wang, G. Bi, and L. Yang, "An autofocus technique for high-resolution inverse synthetic aperture radar imagery," IEEE Transactions on Geoscience and Remote Sensing, vol. 52, no. 10, pp. 6392-6403, 2014.

[3] F. Wen, Z. Zhang, K. Wang, G. Sheng, and G. Zhang, "Angle estimation and mutual coupling self-calibration for ULA-based bistatic MIMO radar," Signal Processing, vol. 144, pp. 61-67, 2018.

[4] J. Li, X. Zhang, R. Cao, and M. Zhou, "Reduced-dimension MUSIC for angle and array gain-phase error estimation in bistatic MIMO radar," IEEE Communications Letters, vol. 17, no. 3, pp. 443-446, 2013.

[5] F. Wen, Z. Zhang, and G. Zhang, "Joint DOD and DOA estimation for bistatic MIMO radar: a covariance trilinear decomposition perspective," IEEE Access, vol. 7, no. 1, pp. 53273-53283, 2019.

[6] F. Wen, X. Xiong, and Z. Zhang, "Angle and mutual coupling estimation in bistatic MIMO radar based on PARAFAC decomposition," Digital Signal Processing, vol. 65, pp. 1-10, 2017.

[7] X.-Y. Pan, W. Wang, and G.-Y. Wang, "Sub-nyquist sampling jamming against ISAR with CS-based HRRP reconstruction," IEEE Sensors Journal, vol. 16, no. 6, pp. 1597-1602, 2016.

[8] X. Zhang, T. Bai, H. Meng, and J. Chen, "Compressive sensing-based ISAR imaging via the combination of the sparsity and nonlocal total variation," IEEE Geoscience and Remote Sensing Letters, vol. 11, no. 5, pp. 990-994, 2014.

[9] L. Ding and W. Chen, "MIMO radar Sparse Imaging with phase mismatch," IEEE Geoscience and Remote Sensing Letters, vol. 12, no. 4, pp. 816-820, 2015. 
[10] F. Q. Wen and J. P. Shi, "Fast direction finding for bistatic EMVS-MIMO radar without pairing," Signal Processing, vol. 173, Article ID 107512, 2020.

[11] L. Wan, G. Han, L. Shu, S. Chan, and T. Zhu, "The application of DOA estimation approach in patient tracking systems with high patient density," IEEE Transactions on Industrial Informatics, vol. 12, no. 6, pp. 2353-2364, 2016.

[12] X. Wang, L. Wan, M. Huang, C. Shen, Z. Han, and T. Zhu, "Low-complexity channel estimation for circular and noncircular signals in virtual MIMO vehicle communication systems," IEEE Transactions on Vehicular Technology, vol. 69, no. 4, pp. 3916-3928, 2020.

[13] L. Wan, G. Han, J. Jiang, J. J. P. C. Rodrigues, N. Feng, and T. Zhu, "DOA estimation for coherently distributed sources considering circular and noncircular signals in massive MIMO systems," IEEE Systems Journal, vol. 11, no. 1, pp. 41-49, 2017.

[14] H. Wang, L. Wan, M. Dong, K. Ota, and X. Wang, “Assistant vehicle localization based on three collaborative base stations via SBL-based robust DOA estimation," IEEE Internet of Things Journal, vol. 6, no. 3, pp. 5766-5777, 2019.

[15] H. Duan, L. Zhang, J. Fang, L. Huang, and H. Li, "Patterncoupled sparse Bayesian learning for inverse synthetic aperture radar imaging," IEEE Signal Processing Letters, vol. 22, no. 11, pp. 1995-1999, 2015.

[16] H. Mohimani, M. Babaie-Zadeh, and C. Jutten, "A fast approach for overcomplete sparse decomposition based on smoothed \$lell $\{0\} \$$ norm," IEEE Transactions on Signal Processing, vol. 57, no. 1, pp. 289-301, 2009.

[17] Y. C. Eldar, P. Kuppinger, and H. Bolcskei, "Block-sparse signals: uncertainty relations and efficient recovery," IEEE Transactions on Signal Processing, vol. 58, no. 6, pp. 30423054, 2010.

[18] R. G. Baraniuk, V. Cevher, M. F. Duarte, and C. Hegde, "Model-based compressive sensing," IEEE Transactions on Information Theory, vol. 56, no. 4, pp. 1982-2001, 2010.

[19] H. S. Chalehjegh, M. Babaie-Zadeh, and C. Jutten, "Fast blocksparse decomposition based on SL0," in Proceedings of the 9th International Conference on Latent Variable Analysis and Signal Separation, Springer, Berlin, Germany, pp. 426-433, September 2010.

[20] E. Van Den Berg and M. P. Friedlander, "Sparse optimization with least-squares constraints," SIAM Journal on Optimization, vol. 21, no. 4, pp. 1201-1229, 2011.

[21] S. S. Chen, D. L. Donoho, and M. A. Saunders, "Atomic decomposition by basis pursuit," SIAM Review, vol. 43, no. 1 , pp. 129-159, 2001.

[22] D. P. Wipf and B. D. Rao, "An empirical Bayesian strategy for solving the simultaneous sparse approximation problem," IEEE Transactions on Signal Processing, vol. 55, no. 7, pp. 3704-3716, 2007. 